\title{
Gain-of-function microRNA screens identify miR-193a regulating proliferation and apoptosis in epithelial ovarian cancer cells
}

\author{
HARUO NAKANO $^{1,2}$, YOJI YAMADA ${ }^{1}$, TATSUYA MIYAZAWA ${ }^{1}$ and TETSUO YOSHIDA $^{1}$ \\ ${ }^{1}$ Biologics Research Laboratories, Kyowa Hakko Kirin Co., Ltd., Machida-shi, Tokyo 194-8533, Japan
}

Received January 31, 2013; Accepted March 19, 2013

DOI: $10.3892 /$ ijo.2013.1896

\begin{abstract}
MicroRNAs (miRNAs) are a small class of non-coding RNAs that negatively regulate gene expression, and are considered as new therapeutic targets for treating cancer. In this study, we performed a gain-of-function screen using miRNA mimic library (319 miRNA species) to identify those affecting cell proliferation in human epithelial ovarian cancer cells (A2780). We discovered a number of miRNAs that increased or decreased the cell viability of A2780 cells. Pro-proliferative and anti-proliferative miRNAs include oncogenic miR-372 and miR-373, and tumor suppressive miR-124a, miR-7, miR-192 and miR-193a, respectively. We found that overexpression of miR-124a, miR-192, miR193a and miR-193b inhibited BrdU incorporation in A2780 cells, indicating that these miRNAs affected the cell cycle. Overexpression of miR-193a and miR-193b induced an activation of caspase $3 / 7$, and resulted in apoptotic cell death in A2780 cells. A genome-wide gene expression analysis with miR-193a-transfected A2780 cells led to identification of ARHGAP19, CCND1, ERBB4, KRAS and MCL1 as potential miR-193a targets. We demonstrated that miR-193a decreased the amount of MCL1 protein by binding 3'UTR of its mRNA. Our study suggests the potential of miRNA screens to discover miRNAs as therapeutic tools to treat ovarian cancer.
\end{abstract}

\section{Introduction}

MicroRNAs (miRNAs) are small non-coding RNAs of 20-22 nucleotides, and function to suppress the expression of target mRNAs by translation blockade and/or mRNA degradation $(1,2)$. They are involved in many biological processes

Correspondence to: Dr Tetsuo Yoshida, Biologics Research Laboratories, Kyowa Hakko Kirin Co., Ltd., 3-6-6, Asahi-machi, Machida-shi, Tokyo 194-8533, Japan

E-mail: tetsuo.yoshida@kyowa-kirin.co.jp

Present address: ${ }^{2}$ Laboratory of Environmental Molecular Physiology, School of Life Sciences, Tokyo University of Pharmacy and Life Sciences, 1432-1, Horinouchi, Hachioji, Tokyo 192-0392, Japan

Key words: microRNA, cell proliferation, apoptosis, miR-193, ovarian cancer cell including cell proliferation, differentiation and apoptosis, and their dysregulation can contribute to the pathological state including cancer $(1,3,4)$. Several groups have documented miRNA expression profiling in ovarian cancers using miRNA microarray and massive parallel sequencing technology (5-10). miR-93, miR-141, miR-200 and miR-214 are frequently upregulated whereas miR-100, miR-143, miR-145 and let-7 are downregulated in ovarian carcinomas compared with normal counterparts (5-9). Abnormal miRNA expression is due to DNA copy number amplification and deletion, epigenetic modification and/or the dysregualtion of miRNA processing in cancer state $(7,11,12)$. miR-214 upregulated in ovarian cancer can target PTEN tumor suppressor gene whereas downregulated let-7 can target the RAS oncogene $(8,13)$, suggesting that miRNAs may have a role as novel class of oncogenes or tumor suppressor genes in ovarian cancer (14).

Based on these findings, the clinical potential of miRNAs as cancer biomarkers and/or therapeutic agents is widely recognized and accepted (15). A single miRNA can regulate multiple mRNA transcripts that cooperatively work in cellular differentiation and function (16-19). The use of miRNA mimics or anti-miRNAs may represent powerful therapeutic tools to accomplish regression and/or re-differentiation of cancer by effectively targeting tumor suppressive or oncogenic genes with less toxicity $(15,20)$. Indeed, a number of pre-clinical trials of miRNAs are currently in progress (21). In this study, we performed a gain of function screen using miRNA mimics library containing 319 miRNAs to identify miRNAs that can affect cell proliferation in A2780 ovary cancer cells. We found several anti-proliferative miRNAs including miR-124, miR-192 and miR-193 in A2780, suggesting that the potential of miRNA screens for discovering miRNAs as therapeutic tools to treat ovarian cancer.

\section{Materials and methods}

Cell culture. Human ovarian cancer cell line A2780 was obtained from Dr T. Tsuruo (22), and human colorectal cancer cell line DLD-1 was obtained from the American Type Culture Collection (ATCC, Manassas, VA, USA). A2780 and DLD-1 were cultured in RPMI-1640 (Gibco, Life Technologies, Carlsbad, CA, USA) containing $50 \mathrm{IU} / \mathrm{ml}$ penicillin and $50 \mu \mathrm{g} / \mathrm{ml}$ streptomycin (Gibco, Life Technologies), supplemented with 5\% (A2780) or $10 \%$ (DLD-1) fetal bovine serum (FBS, JRH Biosciences, Lenexa, $\mathrm{KS}, \mathrm{USA})$ at $37^{\circ} \mathrm{C}$ in an atmosphere of $5 \% \mathrm{CO}_{2}$. 
miRNA library screening. A gain of function miRNA screen on cell viability was performed using A2780 as previously described (23). A2780 was seeded at 2,500 cells per well in 96-well plates the day before transfection. Synthetic miRNA mimic library (human Pre-miR ${ }^{\mathrm{TM}}$ miRNA precursor libraryver.1, Ambion, Applied Biosystems, Foster City, CA, USA) was screened using $50 \mathrm{nM}$ in a duplicate. The library contained 319 miRNAs registered in miRBase ver. 7.1 (http://www.mirbase. org/). miRNA mimics were transfected using Lipofectamine 2000 (Life Technologies) according to the manufacturer's protocols. Pre-miR miRNA precursor molecule-negative control (13) (Ambion, Applied Biosystems) was used as a negative control for miRNA mimics. We confirmed transfection efficiency $(>90 \%)$ using siControl TOX transfection control (50 nM, Dharmacon, Lafayette, CO, USA). After 3 days of transfection, the cell viability was measured using the Cell Titer-Glo Luminescent Cell Viability Assay (Promega, Madison, WI, USA) according to the manufacturer's instructions. Data were expressed as percentage of the negative control. Several miRNA hits were selected to assess reproducibility and dose-dependency $(5,25$ and $50 \mathrm{nM})$.

BrdU incorporation assay. miRNA (25 nM)-transfected cells in 96-well format were harvested for one day, and then were incubated with $10 \mu \mathrm{M}$ of 5'-bromo-2-deoxy-uridine (BrdU) for $2-4 \mathrm{~h}$. The cells were fixed with cold ethanol/ $\mathrm{HCl}$, and the incorporated BrdU was detected using BrdU labeling and detection kit III (Roche Diagnostics GmbH, Mannheim, Germany) according to the manufacturer's instruction.

Caspase 3/7 activation assay. miRNA (25 nM)-transfected cells in 96-well format were harvested for 2 days, and then used to measure caspase 3/7 activity using Caspase-Glo 3/7 assay (Promega) according to the manufacturer's instruction.

RNA isolation and whole genome microarray. A2780 cells were transfected with miR-193a (Pre-miR miRNA precursor molecules, hsa-miR-193a-3p, Ambion, Applied Biosystems) or negative control miRNA $(25 \mathrm{nM})$, and allowed to grow in the medium (RPMI-1640) for $10 \mathrm{~h}$ before RNA isolation. Total RNA was isolated using the RNeasy mini RNA isolation kit (Qiagen). The integrity of the RNA was verified using an Agilent 2100 Bioanalyzer (1.8-2.0: Agilent Technologies, Palo Alto, CA, USA). Transcriptome microarray analysis was carried out using the $44 \mathrm{~K}$ Whole Human Genome Microarray chip (Agilent Technologies) according to the manufacturer's instructions. Scanning microarray chips and processing data were done by Pharmafrontier Co., Ltd, Kyoto, Japan. Differentially expressed probe sets were identified with a fold change $>1.5$. Gene ontology (GO) pathway enrichment analysis was performed among genes differentially expressed after miR-193a transfection by SigTerm software (24). The downregulated genes with miR-193a transfection were compared with predicted miR-193a target genes searched by TargetScan (http://www.targetscan.org/). Over-representation of predicted miR-193a target genes within downregulated gene sets was assessed by SigTerm software.

Western blot analysis. miRNA or siRNA (25 nM)-transfected A2780 cells were lysed in radio immunoprecipitation assay (RIPA) buffer [50 mM Tris- $\mathrm{HCl}$ (pH 8.0), $150 \mathrm{mM}$ sodium chloride, $1 \%$ NP-40, $0.5 \%$ sodium deoxycholate, $0.1 \%$ sodium dodecyl sulfate] supplemented with $1 \%$ of a protease inhibitor cocktail stock solution (set III, Roche Diagnostics GmbH) after 1 or 2 days transfection. The following pre-designed siRNA was used as a positive control: MCL1 siRNA (Hs_MCL1_6 HP validated siRNA, SI02781205, Qiagen GmbH, Hilden, Germany). Proteins (10 or $20 \mu \mathrm{g}$ ) were separated by SDS-PAGE. Upon electroblotting to polyvinylidene fluoride (PVDF) membrane (Immobilon-P, Millipore, Billerica, MA, USA), non-specific binding sites were blocked by incubation in TBST (Tris-buffered saline $/ 0.05 \%$ Tween-20) containing $1 \%$ skim milk, and then incubated with rabbit polyclonal anti-MCL1 (1:200, S-19, Santa Cruz Biotechnology Inc., Santa Cruz, CA, USA), or mouse monoclonal anti- $\alpha$-tubulin $(1: 2,500$, clone DM1A, Sigma, St. Louis, MO, USA) in blocking solution. After washing with TBST, the membrane was incubated with HRP-conjugated rabbit anti-mouse IgG secondary antibody (P0161, Dako, Glostrup, Denmark) or HRP-conjugated swine anti-rabbit IgG secondary antibody (P0217, Dako). Signals were detected using enhanced chemiluminescence (ECL) or ECL-plus reagent Amersham $^{\mathrm{TM}}$ GE Healthcare UK Ltd., Buckinghamshire, UK).

$q R T-P C R$. Total RNA was prepared from miRNA or siRNA (25 $\mathrm{nM})$-transfected cells 2 days after transfection using RNeasy mini kit (Qiagen), and then first strand cDNA was synthesized using SuperScript III (Life Technologies) according to the manufacturer's instruction. Real-time RT-PCR was performed using 7900 HT fast real-time PCR system (Applied Biosystems Inc., Foster City, CA, USA) with SYBR-Green as a reporter. The following primers were used for detection: MCL1 (222 bp) forward: TCTAAGTGCTGAC TGGCTACG, reverse: CCTGGCACAGCTATCAAAAG; GAPDH (137 bp) forward: ACTTTGTCAAGCTCATTTCCTG, reverse: CTCTCTTCCTCTTG GCTCTTG.

Luciferase miRNA target reporter assay. 3'-untranslated regions (UTRs) of MCL1 gene (1546 bp), containing predicted binding sites of miR-193a, were amplified by PCR from A2780 cDNA, and inserted into the pGL3 control vector (Promega) by using Xba-I site immediately downstream from the stop codon of Firefly luciferase. The following primers were used: MCL1 3'-UTR forward: CGGCTAGCGAAAAGCAAGTGG CAAGAGG, reverse: CGGCTAGCAGGGAGGGTCACTCA GGTTT.

Deletion of the first 3 nucleotides corresponding miR-193a seed-region complementary site was inserted in mutant constructs using KOD-plus-Mutagenesis kit (Toyobo, Osaka, Japan), according to the manufacturer's protocol. The following primers were used for generation of mutant constructs: MCL1-mutant-Primer 1: AGCCAGGCAAGTCATAGAATT GATT, MCL1-mutant-Primer 2: GGCCACTTTCCTGTTCT CAACAAGG.

DLD-1 cells were cultured in 96-well formats and co-transfected with $100 \mathrm{ng}$ of pGL3 Firefly luciferase reporter vector, $20 \mathrm{ng}$ of pRL-TK Renilla luciferase control vector (Promega) and $25 \mathrm{nM}$ miRNA or negative control miRNA using Lipofectamine 2000. Firefly and Renilla luciferase activities were measured consecutively using the Dual-Luciferase Reporter Assay System (Promega) $24 \mathrm{~h}$ after transfection. All the experiments were done in triplicate and repeated at least twice on different days. 

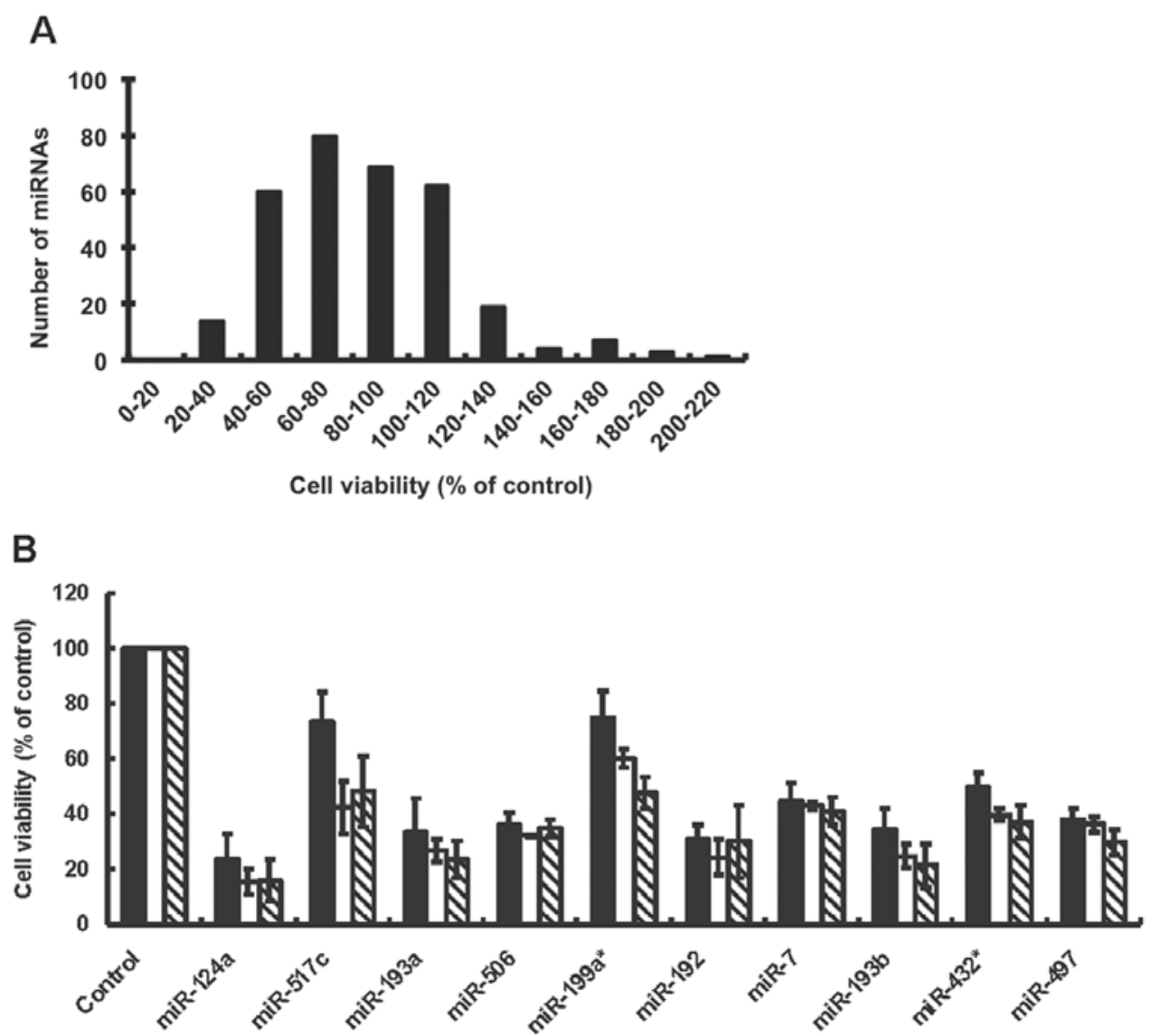

Figure 1. Gain of function screen of miRNA library in A2780 ovary cancer cell line. (A) A2780 cells were transfected with 319 miRNA mimics (50 nM) in duplicate, and their cell viability was measured 3 days after transfection. The graph shows the distribution of cell viability normalized by the miRNA mimic negative control. (B) Top 10 anti-proliferative miRNA mimics derived from 1st screen were transfected into A2780 at the final concentration of $5 \mathrm{nM}$ (solid bar), $25 \mathrm{nM}$ (open bar) and $50 \mathrm{nM}$ (hatched bar). The cell viability was measured three days after miRNA mimic transfection. The data are normalized with the negative control and show average \pm SD from three different experiments.

\section{Results}

Effects of miRNA mimic library transfection on cell proliferation of A2780 cell line. To identify miRNAs that affect cell proliferation of ovarian cancer cells, we performed a gain of function screen using synthetic miRNA mimic library (319 miRNAs) for human epithelial ovary cancer cells (A2780). The library consists of miRNAs registered in early version of miRBase (ver. 7.1 in October, 2005, http://www.mirbase.org/), and many of them were expressed in ovarian normal and cancer tissues and cell lines (5). We detected cellular ATP to assess cell viability in miRNA ( $50 \mathrm{nM}$ )-transfected cells 3 days after transfection. Frequency distribution indicated that broad ranges of miRNA mimic transfections affected the cell viability of A2780 (Fig. 1A). A total of 46 out of 319 miRNAs induced more than $50 \%$ changes in the cell viability of A2780 after 3 days transfection. Table I shows top 10 miRNAs that increased or decreased the cell viability of A2780. They included known oncogenic miRNAs such as miR-372 (cell viability, 187\%) and miR-373 (165\%), and tumor suppressive miRNAs such as miR-124a (28.3\%), miR-7 (37.1\%), miR-192 (36.6\%) and miR-193a (29.7\%) in several different cancer types $(18,25-27)$. The seed family miRNAs that have the same sequences in seed region (2nd to 8th nucleotide) of miRNAs showed similar effects on cell viability
Table I. Results of miRNA library screening.

\begin{tabular}{lcllc}
\hline $\begin{array}{c}\text { miRNAs that increased } \\
\text { cell viability }\end{array}$ & & \multicolumn{2}{c}{$\begin{array}{c}\text { miRNAs that decreased } \\
\text { cell viability }\end{array}$} \\
\cline { 1 - 2 } \cline { 5 - 5 } miRNA & $\begin{array}{c}\text { Cell viability } \\
(\%)\end{array}$ & & miRNA & $\begin{array}{c}\text { Cell viability } \\
(\%)\end{array}$ \\
\hline miR-301 & 218 & & miR-124a & 28.3 \\
miR-372 & 187 & & miR-517c & 29.4 \\
miR-93 & 185 & & miR-193a & 29.7 \\
miR-302b & 181 & & miR-506 & 31.9 \\
miR-130a & 173 & & miR-199a* & 34.5 \\
miR-302d & 172 & & miR-192 & 36.6 \\
miR-363 & 166 & & miR-7 & 37.1 \\
miR-373 & 165 & & miR-193b & 37.7 \\
miR-9* & 162 & & miR-432* & 37.8 \\
miR-130b & 162 & & miR-497 & 38.3 \\
\hline
\end{tabular}

Data represent the cell viability in miRNA mimics $(50 \mathrm{nM})$-transfected cells 3 days after transfection. Data were expressed as a percentage of the negative control in an average of duplicates. Top 10 miRNAs that increased or decreased the cell viability are listed. 



Figure 2. Effects of anti-proliferative miRNAs on DNA synthesis and caspase 3/7 activity. (A) Anti-proliferative miRNAs (miR-124a, miR-192, miR-193a and miR-193b) or negative control miRNA were transfected into A2780 at the final concentration of $25 \mathrm{nM}$. One day after transfection, the transfected cells were incubated with BrdU containing medium for 2 to $4 \mathrm{~h}$, and then BrdU incorporation was measured. The data is normalized with the negative control and show average \pm SD from three different experiments. (B) Anti-proliferative miRNAs (miR-124a, miR-192, miR-193a and miR-193b) or negative control miRNA were transfected into A2780 at the final concentration of $25 \mathrm{nM}$. Two days after transfection, caspase 3/7 activity in miRNA-transfected cells was measured. The data are normalized with the negative control and show average \pm SD from three different experiments. (C) Cellular morphology of miR-193a (25 nM)-transfected A2780 cells. Arrows indicate apoptotic cell debris. Scale bar indicates $100 \mu \mathrm{m}$.

in A2780 cells. For example, miR-93/miR-302/miR-372/mir-373 seed family miRNAs (miR-93, miR-302b, miR-302d, miR-372, miR-373) were pro-proliferative, while miR-193 seed family miRNAs (miR-193a, miR-193b) were anti-proliferative (Table I). miR-200/miR-141 seed family miRNAs that are upregulated in ovarian cancer $(5,6,10)$ had a little effect on the cell viability in A2780 cells (the cell viability; 97.9, 113, 92.0 and $101 \%$ with miR-200a, miR-200b, miR-200c and miR-141 transfection, respectively). miR-100, miR-143 and miR-145 that are downregulated miRNAs in ovarian cancer $(5,6,8)$ induced a $15-30 \%$ decrease in the cell viability of A2780 (the cell viability; 84.1, 81.8 and 73.1 with miR-100, miR-143 and miR-145 transfection, respectively). We are interested in miRNA mimics that decreased the cell viability of A2780 since these miRNA mimics themselves could have therapeutic potential to treat ovarian cancer. To further evaluate miRNA mimics on the inhibition of cell proliferation in A2780, we selected top 10 anti-proliferative miRNAs (miR-7, miR-124a, miR-192, miR-193a, miR-193b, miR-199a*, miR-432*, miR-497, miR-506 and miR-517c) from the first screen, and examined the cell viability in A2780 cells transfected with different concentrations of miRNAs $(5,25$, $50 \mathrm{nM}$ ). We confirmed results of our first screening at $50 \mathrm{nM}$, and found that the transfection of miR-124a, miR-192, miR-193a and miR-193b induced a large decrease in the cell viability of A2780 even at $5 \mathrm{nM}$ (Fig. 1B), indicating that these miRNAs had a profound anti-proliferative effect in A2780 cells. We examined whether miR-124a, miR-192, miR-193a and miR-193b affected DNA synthesis to inhibit cell proliferation in A2780 cells.
One day after miRNA transfection, BrdU incorporation was examined to evaluate DNA synthesis in transfected cells. As shown in Fig. 2A, miR-124a, miR-192, miR-193a and miR-193b decreased an incorporation of BrdU compared with the negative control, indicating that these miRNAs induced the inhibition of DNA synthesis in A2780 cells. We next examined whether these miRNAs affected apoptotic pathway to inhibit cell proliferation in A2780 cells. We found that miR-193a and miR-193b but not miR-124a and miR-192 induced more than twofold increase in an activity of caspase $3 / 7$, the effector of apoptotic pathway, in A2780 cells (Fig. 2B). The result indicated that miR-193a and miR-193b could induce the apoptotic cell death in A2780 cells. Actually, apoptotic cell debris was frequently observed in miR-193a-transfected A2780 cells (Fig. 2C, arrows).

Transcriptome analysis to assess target genes regulated by $m i R-193 a$. We further characterized the anti-proliferative effect of miR-193a in A2780 cells. To examine target genes regulated by miR-193a, we performed genome wide gene expression analysis using miR-193a (25 nM)-transfected cells compared with the negative control miRNA-transfected ones. We identified 518 genes that were downregulated more than 1.5 -fold by miR-193a transfection after $10 \mathrm{~h}$. To evaluate the potential functional significance of the genes downregulated after miR-193a transfection, we subjected the gene expression data to gene ontology (GO) pathway enrichment analysis. The 20 most significantly over-represented pathways listed in Table II include small GTPase signaling and vesicular transport. We compared these 
Table II. Twenty most significantly enriched $(\mathrm{P}<0.05)$ gene ontology (GO) pathways among downregulated genes after miR-193a transfection into A2780 cells.

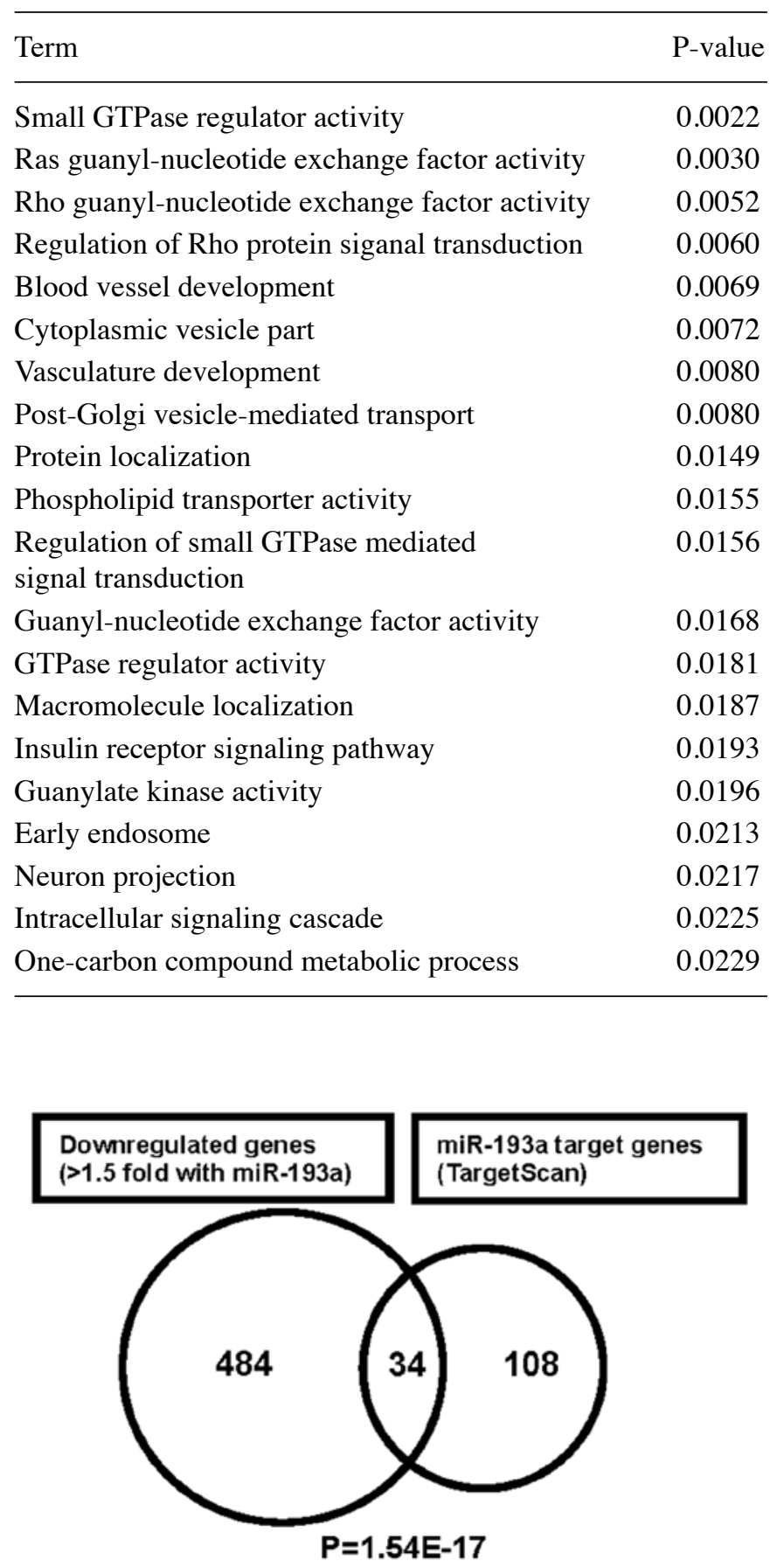

Figure 3. Transcriptome analysis with miR-193a-transfected A2780. Venn diagram to illustrate the relationship between the downregulated genes $(10 \mathrm{~h}$ after miR-193a transfection) and predicted target genes by TargetScan. Predicted miR-193a target genes within the downregulated gene sets were significantly enriched by SigTerm software ( $\mathrm{P}=1.54 \mathrm{E}-17)$.

downregulated 518 genes with predicted miR-193a target genes (142 genes) obtained by TargetScan (Fig. 3). This resulted in the match of 34 candidate miR-193a target genes, and they were significantly over-represented in the downregulated gene sets by using the SigTerm software (24). Table III showed 34 candidate miR-193a target genes obtained by our transcriptome analysis.
Table III. Candidate miR-193a target genes downregulated in miR-193a-transfectants.

\begin{tabular}{|c|c|c|}
\hline Entrez gene ID & Symbol & Fold change \\
\hline 23119 & HIC2 & -6.20 \\
\hline 10152 & $\mathrm{ABI} 2$ & -3.34 \\
\hline 595 & CCND1 & -3.30 \\
\hline 54756 & IL17RD & -3.20 \\
\hline 10238 & WDR68 & -3.15 \\
\hline 3925 & STMN1 & -2.97 \\
\hline 5324 & PLAG1 & -2.97 \\
\hline 3845 & KRAS & -2.76 \\
\hline 4076 & CAPRIN1 & -2.66 \\
\hline 2066 & ERBB4 & -2.58 \\
\hline 57704 & GBA2 & -2.45 \\
\hline 84986 & ARHGAP19 & -2.23 \\
\hline 10620 & ARID3B & -2.17 \\
\hline 7342 & UBP1 & -2.09 \\
\hline 27242 & TNFRSF21 & -2.07 \\
\hline 4170 & MCL1 & -2.00 \\
\hline 56262 & LRRC8A & -1.93 \\
\hline 10160 & FARP1 & -1.91 \\
\hline 57472 & CNOT6 & -1.91 \\
\hline 23179 & RGL1 & -1.79 \\
\hline 23341 & DNAJC16 & -1.79 \\
\hline 88455 & ANKRD13A & -1.70 \\
\hline 4215 & MAP3K3 & -1.67 \\
\hline 114991 & ZNF618 & -1.66 \\
\hline 23492 & CBX7 & -1.64 \\
\hline 23365 & ARHGEF12 & -1.64 \\
\hline 22883 & CLSTN1 & -1.61 \\
\hline 9939 & RBM8A & -1.60 \\
\hline 54890 & ALKBH5 & -1.59 \\
\hline 115 & ADCY9 & -1.57 \\
\hline 4189 & DNAJB9 & -1.51 \\
\hline 1173 & AP2M1 & -1.51 \\
\hline 9962 & SLC23A2 & -1.51 \\
\hline 23384 & SPECC1L & -1.50 \\
\hline
\end{tabular}

The candidate genes include ARHGAP19 (RhoGAP19), CCND1 (cyclin D1), ERBB4, KRAS and MCL1 that function in cell signaling, cell cycle and apoptotic pathway.

MCL1 is a direct target gene of miR-193a in A2780 cells. From our results of transcriptome analysis, we focused on MCL1 gene as miR-193a targets, since MCL1 was an anti-apoptotic gene of BCL2 family (28), and therefore might contribute to miR-193a-induced cell death in A2780 cells. MCL1 3'UTR contains one potential target site of miR-193a and the site is conserved between human and mouse. To examine the regulation of miR-193a on MCL1 protein expression, we performed western blot analysis with miR-193a-transfected A2780 cells. Transfection of positive control MCL1 siRNA induced the decrease in endogenous MCL1 proteins in A2780 cells 
A

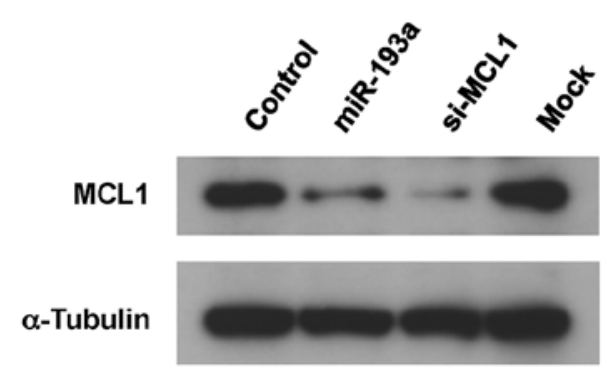

C

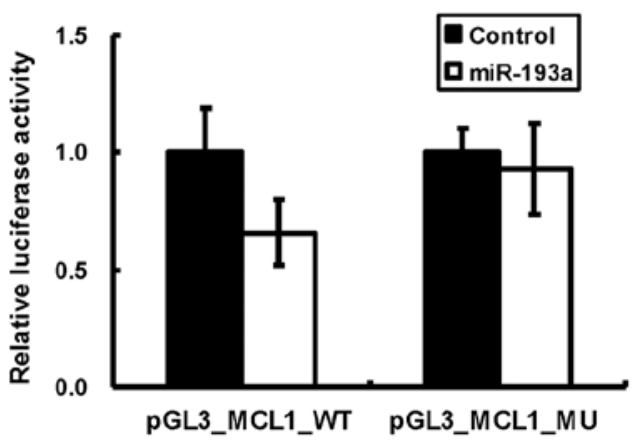

B

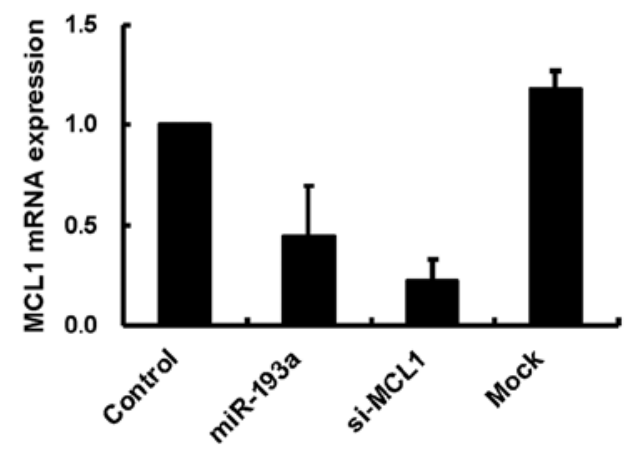

D

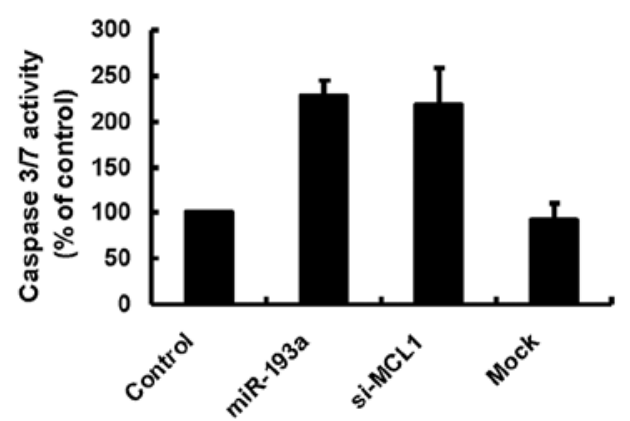

Figure 4. miR-193a targets the anti-apoptotic factor MCL1. (A) Immunoblot analysis for detection of MCL1 with anti-MCL1 antibody. Cell lysate with miR-193a or MCL1 siRNA (25 nM)-transfected cells was collected two days after transfection. Total protein $(10 \mu \mathrm{g})$ was loaded for analysis. The immunodetection of $\alpha$-tubulin was used as loading control. The representative blots of three different experiments are shown. (B) Relative quantification of MCL1 expression by qRT-PCR in miR-193a or MCL1 siRNA (25 nM)-transfected cells two days after transfection. The data are normalized by GAPDH expression, and expressed as relative values against the negative control. Average \pm SD from three different experiments are shown. (C) Reporter assay showing decreased luciferase activity in DLD-1 cells co-transfected with pGL3-MCL1-3'UTR (pGL3_MCL1_WT) and miR-193a (25 nM, open bar). Deletion of miR-193a seed region complementary site from the 3'UTR (pGL3_MCL1_MU) attenuated miR-193a-induced decrease of luciferase activity. Firefly/Renilla luciferase activity is expressed as relative values against the negative control. Average $\pm \mathrm{SD}(\mathrm{n}=3)$ from one representative experiment are shown. (D) Caspase 3/7 activity of miR-193a or MCL-1 siRNA $(25 \mathrm{nM})$-transfected cells was measured 2 days after transfection. The data show average \pm SD from three different experiments.

(Fig. 4A). We demonstrated that overexpression of miR-193a decreased MCL1 proteins in A2780 cells (Fig. 4A). We next performed qRT-PCR with miR-193a-transfected cells to examine whether miR-193a affected MCL1 mRNA expression. We found that miR-193a induced about 50\% decrease in MCL1 mRNA expression in A2780 cells (Fig. 4B). These results indicated that miR-193a affected MCL1 expression at both protein and mRNA levels. To validate whether miR193a can directly regulate the translation of MCL1 mRNAs, we constructed a luciferase reporter plasmid that inserted MCL1 3'UTR (around $1.5 \mathrm{~kb}$ ) at the downstream of Firefly luciferase gene, and tested the luciferase activity. As shown in Fig. 4C, co-transfection of miR-193a and MCL1 3'UTR reporter vector induced around $40 \%$ reduction of the luciferase activity compared with co-transfection of the negative control miRNA and the reporter vector. The decrease of the luciferase activity was attenuated by using the mutant reporter vector deleting miR-193a seed region complementary sites in MCL1
3'UTR (Fig. 4C, MCL1-3'UTR-MU). These results indicated that MCL1 would be a direct target of miR-193a. We further examined whether the downregulation of endogenous MCL1 could induce apoptosis in A2780 cells. As shown in Fig. 4D, the transfection of MCL1 siRNA (25 nM) induced caspase 3/7 activation comparable with miR-193a transfection in A2780 cells (Fig. 4D), indicating that the downregulation of MCL1 by miR-193a could contribute to miR-193a-induced apoptosis in A2780 cells.

\section{Discussion}

Several studies reveal that global miRNA expression is dysregulated in ovarian cancer (5-10), and miRNAs may represent new targets for detection, diagnosis and therapy in ovarian cancer (14). However, functions of many miRNAs in ovarian cancer remain to be elucidated. In this study, we performed a gain-of-function screen using a miRNA mimic library 
(319 miRNA species) to identify those affecting cell proliferation in epithelial ovarian cancer cells (A2780). The library consists of miRNAs registered in early version of miRBase (ver. 7.1 in October, 2005, http://www.mirbase.org/), and many of them were expressed in ovarian normal and cancer tissues and cell lines (5). We discovered pro-proliferative miRNAs (miR-9*, miR-93, miR-130a, miR-130b, miR-301, miR-302b, miR-302d, miR-363, miR-372, miR-373), and anti-proliferative miRNAs (miR-7, miR-124a, miR-192, miR-193a, miR-193b, miR-199a*, miR-432*, miR-497, miR-506, miR-517c) in A2780 cells. By the same miRNA mimics library screening, we found that $\mathrm{miR}-93 / \mathrm{miR}-372 / \mathrm{miR}-373$ and $\mathrm{miR}-124 \mathrm{a}$ were pro-proliferative and anti-proliferative, respectively, in DLD-1 colorectal cancer cells (23), suggesting consistent roles of these miRNAs on cell proliferation in ovary and colorectal cancer cells. The base-pairing between target mRNAs and the seed region (2nd to 8th nucleotides) of miRNA is important for miRNAs to function to regulate their target genes (2). The seed family miRNAs induced similar cellular phenotypes on cell proliferation in this study (ex. pro-proliferative miR-93, miR-302b, miR-302d, miR-372, miR-373 and anti-proliferative miR-193a, miR-193b), supporting the importance of the seed region of miRNA on its function. Our miRNA hits did not always correspond to dysregulated miRNAs reported in ovarian cancer (5-10), but included pro-proliferative miR-93 that was upregulated in primary ovarian carcinomas (6), supporting an oncogenic role of this miRNA in ovarian cancer. Our miRNA hits also included tumor suppressive miR-7, miR-124a, miR-192 and miR-193a in several cancer types $(18,25-27)$, suggesting that these miRNAs could be tumor suppressive in ovarian cancer. Among our miRNA hits, we further characterized miR-124a, miR-192, miR-193a and miR-193b that induced a large decrease in the cell viability of A2780 cells. miR-124a and miR-192 induced a decrease in BrdU incorporation, indicating that these miRNAs affected cell cycle resulting in inhibition of DNA synthesis in A2780 cells. Inhibitory effects of miR-124a and miR-192 on cell cycle gene pathway are reported in several cancer cell lines. miR-124a targets cyclin dependent kinase 6 (CDK6), and thereby inhibits the phosphorylation of retinoblastoma $(\mathrm{Rb})$ in HCT116 cells (29). miR-192 is upregulated by genotoxic stress in HCT116, A549 and U2OS cell lines bearing wild-type p53, and induces the cell cycle arrest by enhancing CDKN1A/p21 expression $(18,30)$.

We showed that miR-193a and miR-193b inhibited BrdU incorporation and induced caspase 3/7 activation in A2780 cells, indicating that these miRNAs could affect cell cycle and apoptotic gene pathways. Our transcriptome analysis with miR-193a-transfected A2780 cells identified ARHGAP19, CCND1, ERBB4, KRAS, MCL1 as potential miR-193a target genes. We demonstrated that the translation of MCL1 proteins was suppressed by miR-193a, suggesting that anti-apoptotic MCL1 wouldbe one of the target genes for miR-193a-induced cell death in A2780. Anti-proliferative and pro-apoptotic functions of miR-193 are reported in several cancer cell lines including MDA-MB-453 (breast cancer), Malme-3M, SKMEL-28, SKMEL-5 (melanoma), HO-1-N-1, HSC-2 (oral squamous cell carcinoma), 22Rv1 (prostate cancer), SK-Hep-1 (hepatocellular carcinoma) and Kasumi-1 (acute myeloid leukemia) (26,31-36). Consistent with our results, CCND1, KRAS and MCL1 are identified as miR-193 target genes $(26,32,33,37)$.
miR-193a gene locus (chromosomal region 17q11.2) has $\mathrm{CpG}$ islands that are hyper-methylated in oral cancer (26) and acute myeloid leukemia (36) compared with normal tissues and cells. miR-193a is downregulated in epithelial ovary cancer compared with normal counterparts (10), but study is needed on whether the miR-193a gene locus is hyper-methylated in ovary cancer.

Exogenous expression of a single miRNA mimic can coordinately regulate gene expression on cellular function, which encourages the therapeutic use of miRNA to direct cancer cell death and/or re-differentiation without undesirable side-effects $(15,20)$. One of the challenges to the therapeutic use of miRNA is to predict precisely molecular consequences induced by modulating cellular miRNAs. Transcriptome analysis by microarray has been widely used for miRNA target identification at the transcription level. Protein-profiling techniques have been applied to miRNA-transfected cells for the identification of miRNA targets at the translational level (38-41).

In summary, we performed a gain-of-function miRNA screen and discovered several miRNAs affecting cell proliferation and death in A2780 ovary cancer cells. Among them, we identified miR-193a as strong anti-proliferative miRNAs in A2780 cells. miR-193a induced the inhibition of DNA synthesis and apoptosis by targeting genes including ARHGAP19, CCND1, ERBB4, KRAS, MCL1, indicating a tumor suppressive role of this miRNA in epithelial ovarian cancer cells. Our study suggests the potential of miRNA screens to discover miRNAs as therapeutic tools to treat ovarian cancer.

\section{Acknowledgements}

We thank Ms. K. Hayama, Ms. I. Taki and Ms. M. Kamigaki for their technical assistance. This research was partly supported by a grant from the New Energy and Industrial Technology Development Organization (NEDO).

\section{References}

1. He L and Hannon GJ: MicroRNAs: small RNAs with a big role in gene regulation. Nat Rev Genet 5: 522-531, 2004.

2. Fabian MR, Sonenberg N and Filipowicz W: Regulation of mRNA translation and stability by microRNAs. Annu Rev Biochem 79: 351-379, 2010.

3. Calin GA and Croce CM: MicroRNA signatures in human cancers. Nat Rev Cancer 6: 857-866, 2006.

4. Garzon R, Calin GA and Croce CM: MicroRNAs in cancer. Annu Rev Med 60: 167-179, 2009.

5. Iorio MV, Visone R, Di Leva G, Donati V, Petrocca F, Casalini P, Taccioli C, Volinia S, Liu CG, Alder H, Calin GA, Menard S and Croce CM: MicroRNA signatures in human ovarian cancer. Cancer Res 67: 8699-8707, 2007.

6. Nam EJ, Yoon H, Kim SW, Kim H, Kim YT, Kim JH, Kim JW and Kim S: MicroRNA expression profiles in serous ovarian carcinoma. Clin Cancer Res 14: 2690-2695, 2008.

7. Zhang L, Volinia S, Bonome T, Calin GA, Greshock J, Yang N, Liu CG, Giannakakis A, Alexiou P, Hasegawa K, Johnstone CN, Megraw MS, Adams S, Lassus H, Huang J, Kaur S, Liang S, Sethupathy P, Leminen A, Simossis VA, Sandaltzopoulos R, Naomoto Y, Katsaros D, Gimotty PA, DeMichele A, Huang Q, Butzow R, Rustgi AK, Weber BL, Birrer MJ, Hatzigeorgiou AG, Croce CM and Coukos G: Genomic and epigenetic alterations deregulate microRNA expression in human epithelial ovarian cancer. Proc Natl Acad Sci USA 105: 7004-7009, 2008.

8. Yang H, Kong W, He L, Zhao JJ, O'Donnell JD, Wang J, Wenham RM, Coppola D, Kruk PA, Nicosia SV and Cheng JQ: MicroRNA expression profiling in human ovarian cancer: miR-214 induces cell survival and cisplatin resistance by targeting PTEN. Cancer Res 68: 425-433, 2008. 
9. Dahiya N, Sherman-Baust CA, Wang TL, Davidson B Shih IeM, Zhang Y, Wood W III, Becker KG and Morin PJ: MicroRNA expression and identification of putative miRNA targets in ovarian cancer. PLoS One 3: e2436, 2008.

10. Wyman SK, Parkin RK, Mitchell PS, Fritz BR, O'Briant K, Godwin AK, Urban N, Drescher CW, Knudsen BS and Tewari M: Repertoire of microRNAs in epithelial ovarian cancer as determined by next generation sequencing of small RNA cDNA libraries. PLoS One 4: e5311, 2009.

11. Zhang L, Huang J, Yang N, Greshock J, Megraw MS, Giannakakis A, Liang S, Naylor TL, Barchetti A, Ward MR, Yao G, Medina A, O'brien-Jenkins A, Katsaros D, Hatzigeorgiou A, Gimotty PA, Weber BL and Coukos G: microRNAs exhibit high frequency genomic alterations in human cancer. Proc Natl Acad Sci USA 103: 9136-9141, 2006

12. Merritt WM, Lin YG, Han LY, Kamat AA, Spannuth WA, Schmandt R, Urbauer D, Pennacchio LA, Cheng JF, Nick AM, Deavers MT, Mourad-Zeidan A, Wang H, Mueller P, Lenburg ME, Gray JW, Mok S, Birrer MJ, Lopez-Berestein G, Coleman RL, Bar-Eli M and Sood AK: Dicer, Drosha, and outcomes in patients with ovarian cancer. N Engl J Med 359: 2641-2650, 2008.

13. Johnson SM, Grosshans H, Shingara J, Byrom M, Jarvis R, Cheng A, Labourier E, Reinert KL, Brown D and Slack FJ: RAS is regulated by the let-7 microRNA family. Cell 120: 635-647, 2005

14. Dahiya N and Morin PJ: MicroRNAs in ovarian carcinomas. Endocr Relat Cancer 17: F77-89, 2010.

15. Garofalo $M$ and Croce CM: microRNAs: Master regulators as potential therapeutics in cancer. Annu Rev Pharmacol Toxicol 51: 25-43, 2011.

16. Linsley PS, Schelter J, Burchard J, Kibukawa M, Martin MM, Bartz SR, Johnson JM, Cummins JM, Raymond CK, Dai H, Chau N, Cleary M, Jackson AL, Carleton M and Lim L: Transcripts targeted by the microRNA-16 family cooperatively regulate cell cycle progression. Mol Cell Biol 27: 2240-2252, 2007.

17. He L, He X, Lim LP, de Stanchina E, Xuan Z, Liang Y, Xue W, Zender L, Magnus J, Ridzon D, Jackson AL, Linsley PS, Chen C, Lowe SW, Cleary MA and Hannon GJ: A microRNA component of the p53 tumour suppressor network. Nature 447: 1130-1134, 2007.

18. Georges SA, Biery MC, Kim SY, Schelter JM, Guo J, Chang AN, Jackson AL, Carleton MO, Linsley PS, Cleary MA and Chau BN: Coordinated regulation of cell cycle transcripts by p53-inducible microRNAs, miR-192 and miR-215. Cancer Res 68: 10105-10112, 2008.

19. Lim LP, Lau NC, Garrett-Engele P, Grimson A, Schelter JM, Castle J, Bartel DP, Linsley PS and Johnson JM: Microarray analysis shows that some microRNAs downregulate large numbers of target mRNAs. Nature 433: 769-773, 2005.

20. Mishra PJ and Merlino G: MicroRNA reexpression as differentiation therapy in cancer. J Clin Invest 119: 2119-2123, 2009.

21. Wahid F, Shehzad A, Khan T and Kim YY: MicroRNAs: synthesis, mechanism, function, and recent clinical trials. Biochim Biophys Acta 1803: 1231-1243, 2010.

22. Tsuruo T, Hamilton TC, Louie KG, Behrens BC, Young RC and Ozols RF: Collateral susceptibility of adriamycin-, melphalanand cisplatin-resistant human ovarian tumor cells to bleomycin. Jpn J Cancer Res 77: 941-945, 1986.

23. Nakano H, Miyazawa T, Kinoshita K, Yamada Y and Yoshida T: Functional screening identifies a microRNA, miR-491 that induces apoptosis by targeting $\mathrm{Bcl}-\mathrm{X}(\mathrm{L})$ in colorectal cancer cells. Int J Cancer 127: 1072-1080, 2010.

24. Creighton CJ, Nagaraja AK, Hanash SM, Matzuk MM and Gunaratne PH: A bioinformatics tool for linking gene expression profiling results with public databases of microRNA target predictions. RNA 14: 2290-2296, 2008.

25. Kefas B, Godlewski J, Comeau L, Li Y, Abounader R, Hawkinson M, Lee J, Fine H, Chiocca EA, Lawler S and Purow B: microRNA-7 inhibits the epidermal growth factor receptor and the Akt pathway and is down-regulated in glioblastoma. Cancer Res 68: 3566-3572, 2008.
26. Kozaki K, Imoto I, Mogi S, Omura $\mathrm{K}$ and Inazawa J: Exploration of tumor-suppressive microRNAs silenced by DNA hypermethylation in oral cancer. Cancer Res 68: 2094-2105, 2008.

27. Voorhoeve PM, le Sage C, Schrier M, Gillis AJ, Stoop H, Nagel R, Liu YP, van Duijse J, Drost J, Griekspoor A, Zlotorynski E, Yabuta N, De Vita G, Nojima H, Looijenga LH and Agami R: A genetic screen implicates miRNA-372 and miRNA-373 as oncogenes in testicular germ cell tumors. Adv Exp Med Biol 604: 17-46, 2007.

28. Nijhawan D, Fang M, Traer E, Zhong Q, Gao W, Du F and Wang X: Elimination of Mcl-1 is required for the initiation of apoptosis following ultraviolet irradiation. Genes Dev 17: 1475-1486, 2003

29. Lujambio A, Ropero S, Ballestar E, Fraga MF, Cerrato C, Setien F, Casado S, Suarez-Gauthier A, Sanchez-Cespedes M, Git A, Spiteri I, Das PP, Caldas C, Miska E and Esteller M: Genetic unmasking of an epigenetically silenced microRNA in human cancer cells. Cancer Res 67: 1424-1429, 2007.

30. Braun CJ, Zhang X, Savelyeva I, Wolff S, Moll UM, Schepeler T, Orntoft TF, Andersen CL and Dobbelstein M: p53-responsive micrornas 192 and 215 are capable of inducing cell cycle arrest. Cancer Res 68: 10094-10104, 2008.

31. Ovcharenko D, Kelnar K, Johnson C, Leng N and Brown D: Genome-scale microRNA and small interfering RNA screens identify small RNA modulators of TRAIL-induced apoptosis pathway. Cancer Res 67: 10782-10788, 2007.

32. Iliopoulos D, Rotem A and Struhl K: Inhibition of miR-193a expression by Max and RXRalpha activates K-Ras and PLAU to mediate distinct aspects of cellular transformation. Cancer Res 71: 5144-5153, 2011.

33. Chen J, Zhang X, Lentz C, Abi-Daoud M, Pare GC, Yang X, Feilotter HE and Tron VA: miR-193b Regulates Mcl-1 in Melanoma. Am J Pathol 179: 2162-2168, 2011.

34. Xu C, Liu S, Fu H, Li S, Tie Y, Zhu J, Xing R, Jin Y, Sun Z and Zheng X: MicroRNA-193b regulates proliferation, migration and invasion in human hepatocellular carcinoma cells. Eur J Cancer 46: 2828-2836, 2010.

35. Rauhala HE, Jalava SE, Isotalo J, Bracken H, Lehmusvaara S, Tammela TL, Oja H and Visakorpi T: miR-193b is an epigenetically regulated putative tumor suppressor in prostate cancer. Int J Cancer 127: 1363-1372, 2010.

36. Gao XN, Lin J, Gao L, Li YH, Wang LL and Yu L: MicroRNA-193b regulates c-Kit proto-oncogene and represses cell proliferation in acute myeloid leukemia. Leuk Res 35: 1226-1232, 2011.

37. Chen J, Feilotter HE, Pare GC, Zhang X, Pemberton JG, Garady C, Lai D, Yang X and Tron VA: MicroRNA-193b represses cell proliferation and regulates cyclin D1 in melanoma. Am J Pathol 176: 2520-2529, 2010.

38. Baek D, Villen J, Shin C, Camargo FD, Gygi SP and Bartel DP: The impact of microRNAs on protein output. Nature 455: 64-71, 2008.

39. Selbach M, Schwanhausser B, Thierfelder N, Fang Z, Khanin R and Rajewsky N: Widespread changes in protein synthesis induced by microRNAs. Nature 455: 58-63, 2008.

40. Leivonen SK, Makela R, Ostling P, Kohonen P,Haapa-Paananen S, Kleivi K, Enerly E, Aakula A, Hellstrom K, Sahlberg N, Kristensen VN, Borresen-Dale AL, Saviranta P, Perala M and Kallioniemi O: Protein lysate microarray analysis to identify microRNAs regulating estrogen receptor signaling in breast cancer cell lines. Oncogene 28: 3926-3936, 2009.

41. Leivonen SK, Rokka A, Ostling P, Kohonen P, Corthals GL, Kallioniemi O and Perala M: Identification of miR-193b targets in breast cancer cells and systems biological analysis of their functional impact. Mol Cell Proteomics 10: M110.005322, 2011. 